\title{
Rinofima: opções cirúrgicas utilizadas no Serviço de Cirurgia Plástica do Hospital Agamenon Magalhães - PE
}

\author{
Rhinophyma: surgical options used at the Service of Plastic Surgery of Hospital \\ Agamenon Magalhães-PE
}

Thiago Campos Costa ${ }^{1}$

Wood Allen Almeida

FIRME $^{1}$

LeOnardo Maciel Ribeiro

DE BRITO

Maurício Beltrão Gama

VIEIRA $^{3}$

Luiz Alberto de Sousa

LEITE $^{4}$

Trabalho realizado no Hospital Agamenon Magalhães, Recife, PE, Brasil.

Artigo submetido pelo SGP (Sistema de Gestão de Publicações) da RBCP.

Artigo recebido: 10/6/2010 Artigo aceito: 3/11/2011

\begin{abstract}
RESUMO
Introdução: A rinofima é uma inflamação crônica dos tecidos do nariz com acometimento de cor, textura e vascularização, apresentando crescimento exofítico irregular e telangiectasias. Objetivo: Levantar dados epidemiológicos de pacientes portadores de rinofima e avaliar os resultados dos diversos tratamentos para esta patologia realizados no Serviço de Cirurgia Plástica do Hospital Agamenon Magalhães (HAM), Recife, PE, Brasil, comparando-os com os tratamentos descritos na literatura. Método: Estudo retrospectivo, baseado na análise de prontuários de 28 pacientes submetidos a tratamento do rinofima, entre março de $2002 \mathrm{e}$ março de 2010. Resultados: A média de idade dos pacientes foi 50,1 anos, sendo 72,72\% do sexo masculino e $17,28 \%$ do sexo feminino. $O$ tratamento mais utilizado foi associação de decorticação/ dermoabrasão e eletrocoagulação, em $96 \%$ dos casos. Foram observadas as seguintes complicações: 1 caso de hipopigmentação, 2 de hiperpigmentação e 1 de cicatriz inestética. Em 3 casos, houve necessidade de mais de uma cirurgia para complementação do tratamento. Nenhum caso de câncer foi verificado nas peças cirúrgicas. O grau de satisfação dos pacientes foi de $100 \%$. Conclusões: A rinofima é uma doença que possui tratamento simples e eficaz, com excelentes resultados cosméticos.
\end{abstract}

Descritores: Rinofima. Nariz/lesões. Doenças nasais. Cirurgia plástica.

\begin{abstract}
Introduction: Rhinophyma is a chronic inflammation of the nose with involvement of color, texture and vascularization, with irregular exophytic growth and telangiectasia. Objective: To analyze epidemiological data of patients with rhinophyma and evaluate the results of various treatments for this disease at the Service of Plastic Surgery of Hospital Agamenon Magalhães, Recife, PE, Brasil, comparing them with the treatments described in the literature. Methods: A retrospective study using analysis of medical records of 28 patients undergoing treatment of rhinophyma, between March 2002 and March 2010. Results: The average patient age was 50.1 years, with $72.72 \%$ male and $17.28 \%$ female. The most common treatment was an association of decortication/ dermabrasion and electrocoagulation, in $96 \%$ of cases. Complications observed: one case of hypopigmentation, two cases of hyperpigmentation and one case of unsightly scar. In three cases, there was need for more surgery to completion of treatment. No cancer was found on surgical specimens. The degree of patient satisfaction was $100 \%$. Conclusion: Rhinophyma is a disease that has simple and effective treatment with excellent cosmetic results.
\end{abstract}

Keywords: Rhinophyma. Nose/injuries. Nose diseases. Plastic surgery.

\footnotetext{
1. Membro Aspirante da Sociedade Brasileira de Cirurgia Plástica (SBCP); Médico Residente do Serviço de Cirurgia Plástica do Hospital Agamenon Magalhães (HAM), Recife, PE, Brasil.

2. Membro Especialista da SBCP; Cirurgião do Serviço de Cirurgia Plástica do HAM, Recife, PE, Brasil.

3. Membro Especialista da SBCP; Preceptor de Residência Médica do do Serviço de Cirurgia Plástica do HAM, Recife, PE, Brasil.

4. Membro Titular da SBCP; Regente do Serviço de Cirurgia Plástica do HAM, Recife, PE, Brasil.
} 


\section{INTRODUÇÃO}

A rinofima é uma inflamação crônica dos tecidos do nariz com acometimento da cor, textura e da vascularização, com crescimento exofítico irregular e a presença de telangiectasias ${ }^{1-9}$. As mais variadas denominações foram dadas a esta doença, relacionando-a ao aspecto de tubérculos ou aos hábitos que se julgavam seus causadores (Figura 1). Termos como nariz em "couve-flor", nariz de "batata" ou nariz do "alcoólatra" foram substituídos por Hebrea, em 1845, por termos derivados do grego, rhino (nariz) e phyma (crescimento), sendo hoje universalmente aceitos ${ }^{7}$. Outros autores a consideram como a forma mais grave da acne rosácea ${ }^{4}$.

Alterações de cor são frequentes, podendo a pele nasal adquirir uma coloração "vinhosa" ou "arroxeada". Com a evolução da doença, o nariz pode assumir uma consistência firme e, em casos mais avançados, observa-se lobulação do tumor. Casos extremos, com acometimento do mento, bochechas e lóbulos auriculares, já foram descritos.

As alterações se dão ao nível dos vasos da derme, que quando congestos causam hipertrofia e hiperplasia das glândulas sebáceas do nariz.

As cartilagens nasais e áreas internas do nariz não são acometidas histologicamente, porém por ação mecânica podem ser afetadas e necessitarem de correção cirúrgica.

Sua causa ainda não foi estabelecida, mas é sabido que homens brancos, com idade superior a 40 anos, etilistas, com história familiar positiva e com antecedente de exposição solar acentuada são os mais comumente afetados. Além disso, é descrita relação com o consumo de condimentos, cafeína, alimentos quentes e outros fatores que produzam rubor facial. A exposição solar é implicada diretamente no surgimento da rosácea, sendo considerada um fator favorecedor à rinofima. Nesse ponto há controvérsia, pois a acne rosácea é mais comum em mulheres (3:1), em detrimento da rinofima, que predomina em homens. Há indícios de hereditariedade, com descrição de casos ocorridos em gêmeos e relato de incidência de $25 \%$ em tios ou avós dos pacientes acometidos.

Além do caráter estético da reparação, é importante ressaltar a possibilidade de coexistência de câncer no mesmo sítio anatômico, reforçando a necessidade de seu tratamento ${ }^{1,6}$.

O diagnóstico diferencial deve ser feito com leucemia (lesões específicas da pele), lupus eritematoso sistêmico, micose fungoide e acne rosácea graus $1 \mathrm{e} 2$.

Em relação ao histórico do tratamento cirúrgico da rinofima, merecem destaque von Langenbeck, que em 1851 descreveu ressecção com cicatrização por segunda intenção; Ollier, que em 1876 relatou a técnica de decorticação; Wood, que em 1912 realizou enxerto de pele parcial; e

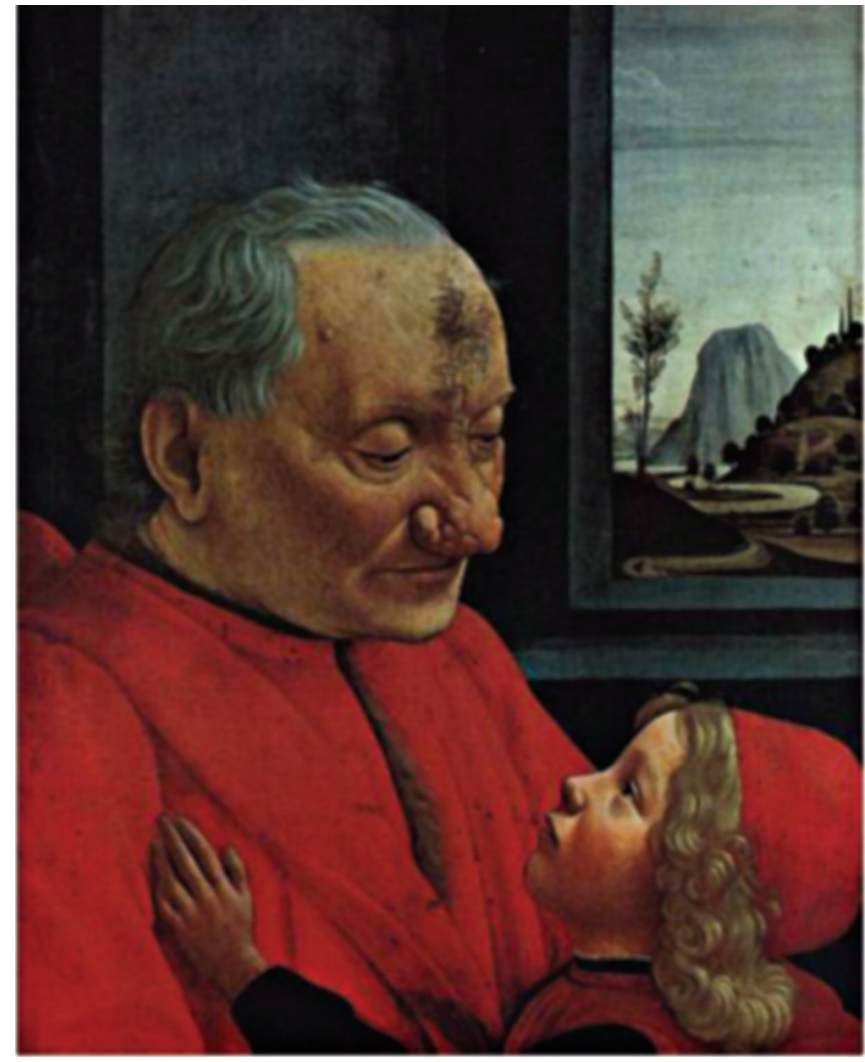

Figura 1 - Ilustração de rinofima pelo célebre pintor florentino Ghirlandaio, no seu famoso quadro "O velho e seu neto", hoje no Museu de Louvre.
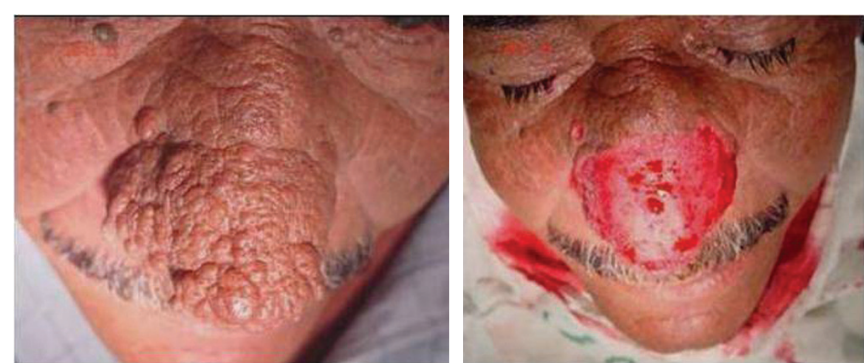

Figura 2 - Tratamento cirúrgico mais utilizado: decorticação + dermoabrasão.
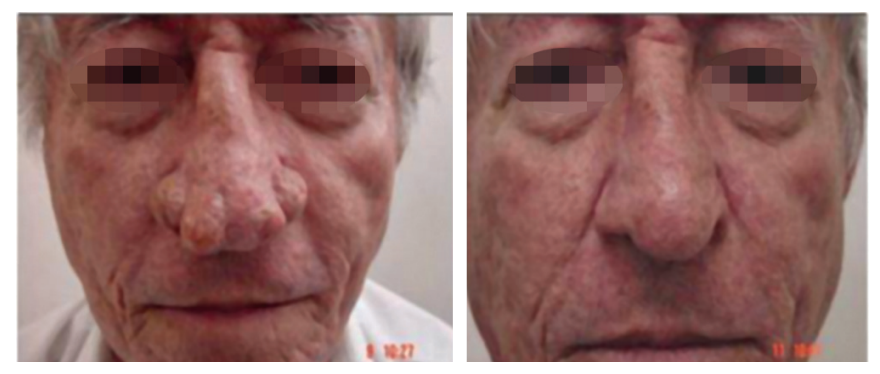

Figura 3 - Paciente 1. Pré-operatório e aspecto após 6 meses do procedimento. 

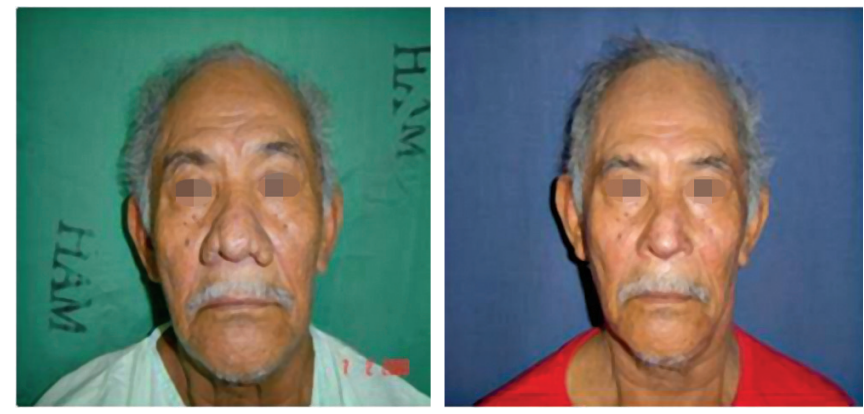

Figura 4-Paciente 2. Pré-operatório e aspecto após 6 meses do procedimento.

Adamopoulous, que em 1961 divulgou a dermoabrasão. Também há relatos do uso de criocirurgia por Nolan, em 1973, e do laser de $\mathrm{CO}_{2}$ por Bohigian, em 1988.

O objetivo deste trabalho é avaliar os resultados dos diversos tratamentos realizados no serviço para o manejo da rinofima. Constatar se, como na literatura, é a dermoabrasão/ eletrocoagulação técnica simples, de bons resultados e poucas complicações. Verificar se, em nosso meio, é uma doença predominantemente masculina e qual a prevalência de cada sexo e, por fim, verificar a incidência de câncer nas peças examinadas.

\section{MÉTODO}

Foi realizado um estudo tipo série de casos, retrospectivo, por meio de análise de prontuários de 28 pacientes submetidos a tratamento do rinofima, no período de março de 2002 e março de 2010, no Hospital Agamenon Magalhães, Recife, PE, Brasil.

Foram analisadas as variáveis sexo, idade, raça, tratamento empregado (modalidade terapêutica/anestesia), resultados obtidos, complicações e incidência de câncer.

Os pacientes foram operados em decúbito dorsal, com anestesia local e sedação ou anestesia geral. Após a antissepsia e assepsia com campos estéreis, foi realizada infiltração do nariz com solução composta por $20 \mathrm{ml}$ de xilocaína a $2 \%$, $5 \mathrm{ml}$ de bicarbonato e $1 \mathrm{ml}$ de adrenalina diluídos em $75 \mathrm{ml}$ solução fisiológica $0,9 \%$.

Após 12 minutos de espera, para que o efeito vasoconstrictor da adrenalina prevalecesse, foi realizada decorticação com lâmina fria $\mathrm{n}^{\circ} 22$ para remoção do tecido hiperplásico, tomando cuidado para não se atingir estruturas nobres, como as cartilagens. A seguir, foi feita dermoabrasão com lixa d'água $\mathrm{n}^{\mathrm{o}} 100$ e para refinamento do contorno nasal com lixa $\mathrm{n}^{\circ} 180$, que também pode ser realizada com dermoabrasão mecânica. Por fim, a revisão rigorosa da hemostasia foi realizada com eletrocoagulação.

A associação de decorticação/ dermoabrasão (Figura 2) e eletrocoagulação foi realizada em 27 (96\%) pacientes. Em 1 (4\%) caso excepcional, foi realizada ressecção seguida de enxertia de pele total.

\section{RESULTADOS}

Dentre os pacientes analisados, $20(72,72 \%)$ eram do sexo masculino e $8(17,28 \%)$ do sexo feminino.

A idade dos pacientes variou entre 40 e 73 anos, com média de 50,1 anos; $40 \%$ dos pacientes eram caucasianos e $60 \%$, pardos.

Os pacientes foram submetidos à anestesia local com sedação em $92 \%$ dos casos e, em $8 \%$ casos, com anestesia geral.

As complicações observadas neste grupo de doentes foram: 1 caso de hipopigmentação (enxerto), 2 casos de hiperpigmentação (decorticação/dermoabrasão) e 1 caso de cicatriz inestética (enxerto).

Em $3(89 \%)$ pacientes, houve necessidade de mais de uma cirurgia para complementação do tratamento, pois se tratavam de casos extremos.

Nenhum caso de câncer foi verificado nas peças cirúrgicas.

O grau de satisfação dos pacientes foi de $100 \%$, após revisão cirúrgica dos 3 casos que necessitaram reintervenção.

As Figuras 3 e 4 ilustram dois casos que constituem a presente casuística.

\section{DISCUSSÃO}

Não foi constatada neoplasia em nenhum dos nossos pacientes. Alguns autores como Churchil e Converse relatam a coexistência desta patologia com o carcinoma basocelular. Segundo Mathes et al. ${ }^{7}$, cerca de 15 a $30 \%$ dos espécimes estudados têm carcinoma basocelular inicial na peça. Converse ${ }^{4}$ afirma, ainda, que existe correlação com o carcinoma espinocelular.

Os casos iniciais podem ser tratados apenas com dermoabrasão. Já em casos avançados, realizamos decorticação cirúrgica, seguida de dermoabrasão para refinamento da ponta.

Houve predominância em pacientes pardos, o que pode ser explicado pela intensa mistura de raças presente na nossa região e menor predominância da população caucasiana.

Em concordância com a literatura, observamos que a decorticação/dermoabrasão é técnica simples, com excelentes resultados e baixo índice de complicações.

Durante a cirurgia, deve-se sempre evitar a exposição de cartilagens, visto que não há acometimento das estruturas profundas do nariz e para não haver comprometimento do resultado estético.

Não utilizamos tratamentos mais agressivos, como retalhos locais, pois entendemos que, para uma patologia benigna, trata-se de uma conduta extremamente agressiva e comprometedora do resultado. 
Nos casos mais graves, extremos, como conduta excepcional, podemos lançar mão de enxerto de pele total.

\section{CONCLUSÕES}

A rinofima é uma doença que possui tratamento simples e eficaz, com excelentes resultados cosméticos.

Como o nariz ocupa a região central da face, é a região mais importante para sua harmonia, assim, o tratamento da rinofima traz grande satisfação aos pacientes, além de quebrar os estigmas da doença.

Embora possa ser necessário mais de um tempo cirúrgico, a recorrência é extremamente rara.

É realizado exame anatomopatológico para pesquisa de malignidade.

Deve-se evitar utilizar alternativas cirúrgicas agressivas.

\section{REFERÊNCIAS}

1. Curnier A, Choudhary S. Triple approach to rhinophyma. Ann Plast Surg. 2002;49(2):211-4.

2. Zanini AS, Carreirão S, Lessa S. Cirurgia do nariz. Rinologia e rinoplastia: funcional, reparadora e estética. Rio de Janeiro:Revinter;1994. p.89-94.

3. Cohen AF, Tiemstra JD. Diagnosis and treatment of rosacea. J Am Board Fam Pract. 2002;15(3):214-7.

4. Converse JM. Reconstructive plastic surgery. vol. 2. Philadelphia: W. B. Saunders;1977. p.1185-92.

5. Franco T. Princípios de cirurgia plástica. São Paulo:Atheneu;2002. p.428.

6. Gunter JP, Rohrich RJ, Adams WP Jr. Dallas rhinoplasty: nasal surgery by the masters. 1st ed. St. Louis:Quality Medical Publishing;2002.

7. Mathes SJ, Weinberger SE, Hentz VR. Plastic surgery: tumors of head, neck and skin. vol. 5. $2^{\mathrm{a}}$ ed. Philadelphia: W. B. Saunders;2006. p.254.

8. Smith JW, Aston S. Grabb and Smiths plastic surgery. 4th ed. Boston:Litle Brown;1991. p.841-2.

9. Stark RB. Plastic surgery of the head and neck. vol. 1. New York: Churchill Livingstone; 1987. p.595-600.

Correspondência para:

Luiz Alberto de Sousa Leite

Hospital Agamenon Magalhães - HAM Serviço de Cirurgia Plástica

Estrada do Arraial, 2723 - Casa Amarela - Recife, PE, Brasil - CEP: 52010-040

E-mail: leitela@hotmail.com 